\title{
Vitreous Hemorrhage, CTCAE 5.0
}

National Cancer Institute

\section{Source}

National Cancer Institute. Vitreous Hemorrhage, CT CAE 5.0. NCI Thesaurus. Code

C146677.

A disorder characterized by bleeding into the vitreous humor. 